Journal of Computer Science 3 (8): 633-638, 2007

ISSN 1549-3636

(C) 2007 Science Publications

\title{
An Innovative Technique of Texture Classification and Comparison Based on Long Linear Patterns
}

\author{
${ }^{1}$ V.Vijaya Kumar, ${ }^{2}$ B.Eswara Reddy, ${ }^{3}$ U.S.N.Raju, ${ }^{4}$ K.Chandra Sekharan \\ ${ }^{1,3}$ Dept. of CSE and IT, GIET, Rajahmundry, A.P., India \\ ${ }^{2}$ Associate Professor in CSE, JNTU College of Engineering, Anantapur, A.P., India \\ ${ }^{4}$ Professor of CSE, National Institute of Technology, Surathkal, Karnataka, India
}

\begin{abstract}
The present paper proposes a method of texture classification based on long linear patterns. Linear patterns of long size are bright features defined by morphological properties: linearity, connectivity, width and by a specific Gaussian-like profile whose curvature varies smoothly along the crest line. The most significant information of a texture often appears in the occurrence of grain components. That's why the present paper used sum of occurrence of grain components for feature extraction. The features are constructed from the different combination of long linear patterns with different orientations. These features offer a better discriminating strategy for texture classification. Further, the distance function captured from the sum of occurrence of grain components of textures is expected to enhance the class seperability power. The class seperability power of these features is investigated in the classification experiments with arbitrarily chosen texture images taken from the Brodatz album. The experimental results indicated good analysis, and how the classification of textures will be effected with different long linear patterns.
\end{abstract}

Keywords: Orientations, Linearity, Connectivity, Features.

\section{INTRODUCTION}

Texture classification is an image processing technique by which different regions of an image are identified based on texture properties. This process plays an important role in many areas such as industrial automation, biomedical image processing, Content Based Image Retrieval and remote sensing application. In spite of the importance of textures in many areas of image processing, there is no universally accepted definition for the texture. We prefer to adopt the definition suggested in ${ }^{[1]}$, because of its generality and it is given as follows: "The notion of texture appears to depend upon three ingredients: (i) some local 'order' is repeated over a region which is large in comparison to the order's size, (ii) the order consists in the nonrandom arrangement of elementary parts, and (iii) the parts are roughly uniform entities having approximately the same dimensions everywhere within the textured region".

This definition explains that the texture is characterized not only by gray value at a given pixel, but also by the gray value pattern in the surrounding pixels. The texture has both local and global meaning, in the sense that it is characterized by the invariance of certain local attributes that are distributed over a region of an image ${ }^{[2]}$. To design an effective algorithm for texture classification, it is essential to find a set of texture features with good discriminating powers. Most of the textural features are generally obtained from the application of a local operator, statistical analysis, or measurement in a transformed domain. Generally, the features are estimated from co-occurrence matrices, Law's texture energy measures, Fourier transform domain, Markov random field models, local linear transforms etc. A number of texture classification techniques are reported in literature $[3,4,5,6]$. The wavelet methods ${ }^{[3,6,7]}$ offer computational advantages over other methods for texture classification and segmentation. In ${ }^{[8]}$, Haralick features ${ }^{[9]}$ are obtained from wavelet decomposed image yielding improved classification rates. In ${ }^{[10]}$, texture features are characterized by considering intensity and contextual information obtained from binary images. The conditional co-occurrence histograms are computed

Corresponding Author: Dr. V. Vijaya Kumar, Dean and Professor of CSE \& IT, Godavari Institute of Engineering and Technology, N.H.-5, Chaitanya Nagar, Rajamundary, East Godavari (Dist), Andhra Pradesh, India. Pin: 533 294. Tel.:+91-9849452287 
from the intensity and binary images. To obtain binary images the fixed thresholds were used. It is evident that the context or the position information of a pixel in an image is very important for the purpose of classification.

In this, paper, we propose a novel scheme of texture classification based on sum of occurrences of grain components in long linear patterns (LLP) with different orientations.

This paper is organized as follows: In section 2, we briefly review theory of linear patterns. The methodology of feature extraction and distance function are evaluated in section 3. The analysis on texture classification based on experimental results is presented in section 4. Concluding remarks are given in section 5 .

\section{LONG LINEAR PATTERNS (LLP)}

The linear elements considered in the present paper are long one-dimensional line elements or patterns or structuring elements. The one-dimensional LLP play a significant role in many image processing operations, such as segmentation, edge detection, classification etc. Moreover line structuring elements are more suited for many morphological operations. In some image processing applications, the square, hexagon, and octagon patterns are used. However the above patterns can be easily decomposed into two, three and four line segments respectively ${ }^{[11,12]}$. The advantage of using one dimensional line pattern segments instead of $\mathrm{N}$ dimensional structuring elements is a reduction in the computational complexity.

In the present paper LLP on the texture images are computed basically by using Bresenham's line algorithm ${ }^{[13]}$. Bresenham ${ }^{[13]}$ published an algorithm to draw a line segment of any size and of any orientation on a plotter, which could draw horizontal, vertical and diagonal lines. The algorithm combines small portions of these lines to form a line pattern of any orientation. In image processing, Bresenham lines are formed by steps in the eight cardinal directions of the grid. But true line patterns can be formed by using Bresenham's line algorithm, for the lines on $\mathrm{X}$ axis, $\mathrm{Y}$ axis and $\mathrm{X}=\mathrm{Y}$ axis, i.e. the lines with $0^{0}, 180^{\circ}, 90^{\circ}, 270^{\circ}$ $, 45^{0}, 135^{0}, 225^{0}, 315^{0}$. For all other orientation, the shape of the LLP appears as steps of a stair case, known as Jaggies.

The LLPs of size $1 \times 11$ with $0^{\circ}, 15^{\circ}, 30^{\circ}$ and $45^{\circ}$ orientations are shown in the following Fig. 1(a), 1(b), $1(c)$ and $1(d)$ respectively. The following figures clearly indicate that the LLP length, defined by an integer number of pixels, depends on the degree of orientation. Each degree of orientation of the LLP will have a

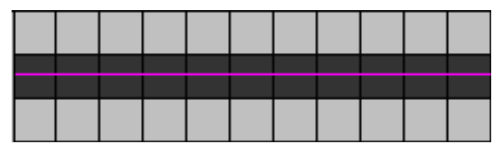

(a)

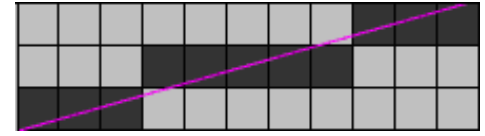

(b)

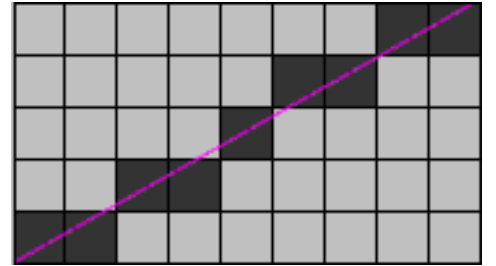

(c)

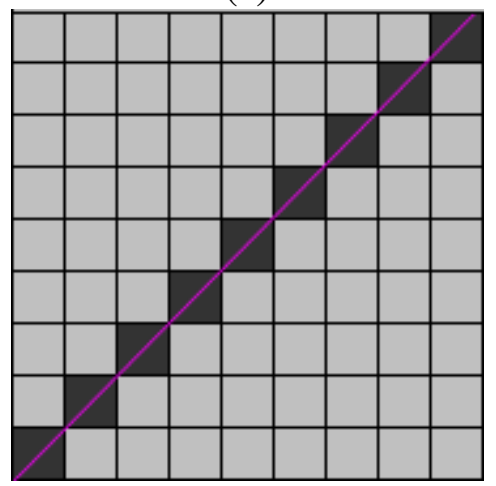

(d)

Fig. 1: LLP of Size $1 \times 11$ (a) $0^{0}$ orientation. (b) $15^{0}$ orientation. (c) $30^{\circ}$ orientation. (d) $45^{\circ}$ orientation.

different set of lengths. The present paper assumes the orientation of LLP as triangular shape shown in Fig.2, but not as a semi circle. This is one of the reasons why

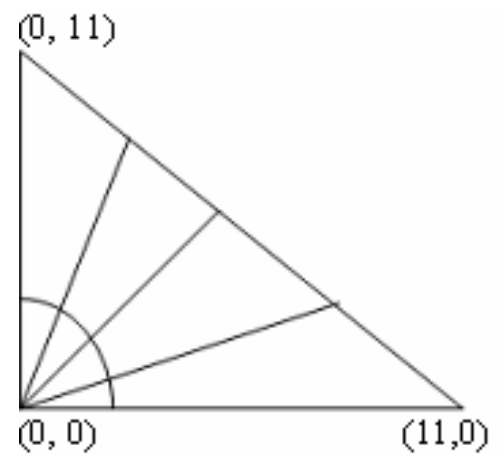

Fig. 2: The structure of long linear pattern with different orientations. 
length of the LLP is reduced for orientations other than $\mathrm{X}$ axis and $\mathrm{Y}$ axis. For example the LLP $1 \mathrm{x} 11$, is having length $11,11,9$ and 9 for $0^{\circ}, 15^{\circ}, 30^{\circ}$ and $45^{\circ}$ orientations respectively. The length is decreased for $30^{\circ}$ and $45^{\circ}$ orientation because of the triangular orientation as shown in Fig.2. Where as the exact length of 11 pixels are resulted for the $0^{0}$ and $15^{\circ}$ orientation, as shown in the Fig.1 (a) and 1(b) respectively. This is because $0^{0}$ orientation falls on X-axis and $15^{\circ}$ orientation is very closer to $\mathrm{X}$-axis.

\section{METHODOLOGY}

The present paper is not concentrated in studying the frequency of occurrence of one dimensional LLP on the texture image because their frequency occurrences will be minimum (most of the times one digit). The classification may become a problem if the frequency count is low. Moreover the most significant information of a texture appears in the occurrence of grain components. That's why the present paper had chosen the sum of occurrence of grain components of LLP as feature extraction. For this one dimensional LLP's of different sizes $1 \times 11,1 \times 13,1 \times 15$, and $1 \times 17$ are chosen. The above LLP's are rotated for every $15^{\circ}$ from $0^{0}$ to $90^{\circ}$. The change of topology on LLP with respect to summation of number of occurrences, of grain components are counted, as shown in Fig.1. This experiment is carried out on thirteen Brodatz textures and is listed from Table 3 to Table 6. In order to classify the textures a distance function is used in the present paper. The distance function is calculated in the following way.

Table 1: Distance function with orientations.

\begin{tabular}{ccccc}
\hline Name of Texture & $\mathrm{GX}_{1}{ }^{\mathrm{o}}$ & $\mathrm{GX}_{2}{ }^{\mathrm{o}}$ & ---- & $\mathrm{GX}_{\mathrm{n}}{ }^{\mathrm{o}}$ \\
\hline $\mathrm{T}_{1}$ & $\mathrm{~A}_{1}$ & $\mathrm{~A}_{2}$ & ---- & $\mathrm{A}_{\mathrm{n}}$ \\
$\mathrm{T}_{2}$ & $\mathrm{~B}_{1}$ & $\mathrm{~B}_{2}$ & ---- & $\mathrm{B}_{\mathrm{n}}$
\end{tabular}

Distance between

Textures with same $\quad\left|A_{1}-B_{1}\right| \quad\left|A_{2}-B_{2}\right| \quad----\left|A_{n}-B_{n}\right|$ orientations.

$$
D\left(T_{1}, T_{2}\right)=\sum_{i=1}^{n}\left(A B S\left(A_{i}-B_{i}\right)^{2}\right)
$$

where $\mathrm{GX}_{1}{ }^{\mathrm{o}}$ represents the total number of occurrences of grain components by $\mathrm{X}_{\mathrm{i}}^{\mathrm{o}}$, of texture $\mathrm{T}_{\mathrm{k}}$. And $\mathrm{D}\left(\mathrm{T}_{1}\right.$, $\mathrm{T}_{2}$ ) is the absolute overall difference between the textures $\mathrm{T}_{1}$ and $\mathrm{T}_{2}$.
The entire process of enumerating the classification of texture is listed in the Fig. 3.

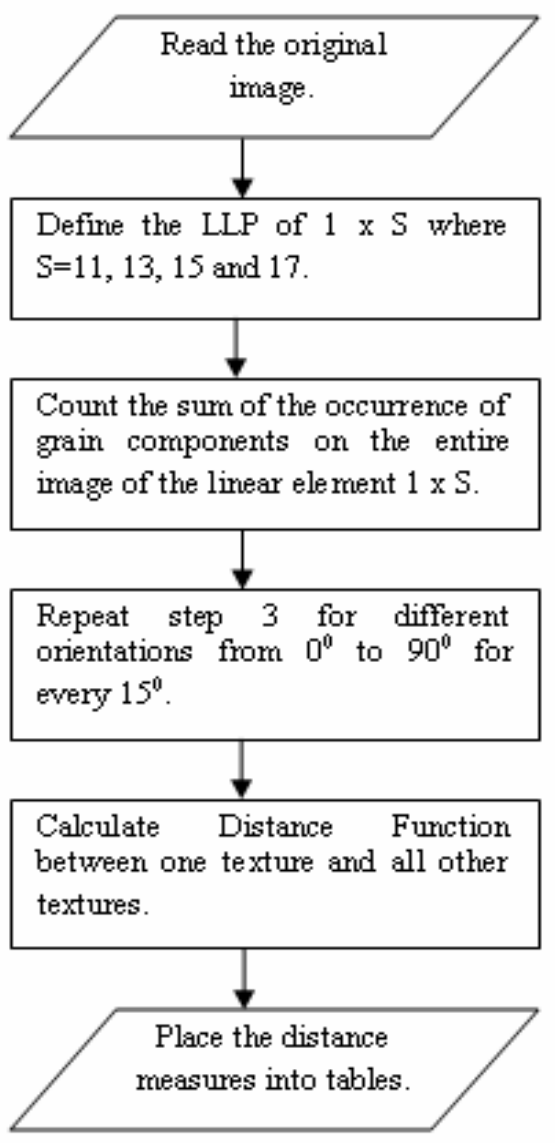

Fig. 3: Block diagram of entire process.

Table 2: Brodatz Texture.

\begin{tabular}{cc}
\hline Texture & Brodatz Texture name \\
\hline $\mathrm{T}_{1}$ & Bark(D12) \\
$\mathrm{T}_{2}$ & Beach sand(D29) \\
$\mathrm{T}_{3}$ & Brick wall(D94) \\
$\mathrm{T}_{4}$ & Grass(D9) \\
$\mathrm{T} 5$ & Herringbone weave(D15) \\
$\mathrm{T}_{6}$ & Pigskin(D92) \\
$\mathrm{T}_{7}$ & Plastic bubbles(D112) \\
$\mathrm{T}_{8}$ & Pressed calf leather(D24) \\
$\mathrm{T}_{9}$ & Raffia(D84) \\
$\mathrm{T}_{10}$ & Straw(D15) \\
$\mathrm{T}_{11}$ & Water(D38) \\
$\mathrm{T}_{12}$ & Wood grain(D68) \\
$\mathrm{T}_{13}$ & Woolen cloth(D19) \\
\hline
\end{tabular}




\section{RESULTS AND ANALYSIS}

RESULTS: The above scheme of classification is applied on randomly chosen 13 Brodatz textures as given in Table 2. Here on wards the texture number is presented instead of texture name. The Tables 3, 4, 5 and 6 gives the sum of grain components of LLP 1x11, $1 \times 13,1 \times 15$ and $1 \times 17$ respectively for all 13 textures with orientations ranging from $0^{0}$ to $90^{0}$ for every $15^{0}$.

ANALYSIS: The Tables 7, 8, 9 and 10 indicate the distance measure between all thirteen textures in all orientations, of LLP of size 1x11, 1x13, 1x15 and 1x17 respectively. The diagonal elements of all distance Tables 7, 8, 9 and 10 prove the following fact, that the distance between same textures is zero. i.e. DIST $\left(T_{i}\right.$, $\left.\mathrm{T}_{\mathrm{i}}\right)=0$.
The textures that differ with a distance threshold factor of ' $d$ ' can be considered as one class. That is two or more textures can be placed into one class ' $\mathrm{C}$ ' if each texture differs with all other textures in the group by a distance of less than or equal to $d$, as specified below.

$C=\left\{T_{i}, T_{i+1}, T_{i+2},----, T_{n}\right\}$, this is true if and only if for all textures, $\mathrm{D}\left(\mathrm{T}_{\mathrm{i},}, \mathrm{T}_{\mathrm{j}}\right)<=\mathrm{d}$, where $\mathrm{i}, \mathrm{j}$ are 1 to $\mathrm{n}$ and $\mathrm{i} \neq \mathrm{j}$.

The following analysis on classification of textures for all LLP's has been done with the same distance threshold value 20. Careful analysis of Table 7, of LLP $1 \mathrm{x} 11$ reveals the following texture classification for a unique distance threshold value, 20.

$\mathrm{C}_{1}=\left\{\mathrm{T}_{1}, \mathrm{~T}_{2}, \mathrm{~T}_{3}, \mathrm{~T}_{10}, \mathrm{~T}_{12}\right\}<=\mathrm{d}_{\mathrm{i}}$

$\mathrm{C} 2=\left\{\mathrm{T}_{4}, \mathrm{~T}_{5}, \mathrm{~T}_{7}, \mathrm{~T}_{8}, \mathrm{~T}_{9}, \mathrm{~T}_{11}, \mathrm{~T}_{13}\right\}<=\mathrm{d}_{\mathrm{i}}$ $\mathrm{C} 3=\left\{\mathrm{T}_{6}\right\}$

Table 3: Sum of occurrence of grain components for LLP of size 11

\begin{tabular}{|c|c|c|c|c|c|c|c|c|c|c|c|c|c|}
\hline & $\mathrm{T} 1$ & $\mathrm{~T} 2$ & T3 & $\mathrm{T} 4$ & T5 & T6 & T7 & T8 & T9 & T10 & T11 & T12 & T13 \\
\hline SEO $0^{\circ}$ & 247,518 & 240,465 & 248,436 & 221,497 & 226,064 & 272,382 & 209,561 & 216,544 & 213,224 & 233,622 & 216,200 & 247,917 & 218,532 \\
\hline SE $15^{\circ}$ & 245,853 & 238,906 & 246,746 & 220,147 & 224,614 & 270,653 & 208,127 & 215,157 & 211,773 & 232,168 & 215,127 & 246,095 & 216,782 \\
\hline $\operatorname{SE} 30^{\circ}$ & 201,351 & 195,778 & 202,112 & 180,453 & 183,913 & 222,417 & 170,377 & 176,198 & 173,283 & 190,236 & 176,059 & 201,757 & 177,752 \\
\hline $\mathrm{SE} 45^{\circ}$ & 197,506 & 191,895 & 198,670 & 177,090 & 180,488 & 219,626 & 167,110 & 172,747 & 169,967 & 186,710 & 172,755 & 197,405 & 174,755 \\
\hline SE $60^{\circ}$ & 199,761 & 194,006 & 200,118 & 179,066 & 182,296 & 221,964 & 168,826 & 174,429 & 171,779 & 188,600 & 174,417 & 200,121 & 176,389 \\
\hline SE $75^{\circ}$ & 247,007 & 239,728 & 246,198 & 221,634 & 224,755 & 275,278 & 208,660 & 214,834 & 211,905 & 232,854 & 215,523 & 248,526 & 217,687 \\
\hline SE $90^{\circ}$ & 249,649 & 242,514 & 249,630 & 224,138 & 227,081 & 278,198 & 211,234 & 216,804 & 213,663 & 235,236 & 217,672 & 252,207 & 219,635 \\
\hline
\end{tabular}

Table 4: Sum of occurrence of grain components for LLP of size 13

\begin{tabular}{|c|c|c|c|c|c|c|c|c|c|c|c|c|c|}
\hline & $\mathrm{T} 1$ & $\mathrm{~T} 2$ & T3 & $\mathrm{T} 4$ & T5 & T6 & $\mathrm{T} 7$ & T8 & T9 & $\mathrm{T} 10$ & $\mathrm{~T} 11$ & $\mathrm{~T} 12$ & T13 \\
\hline $\mathrm{SEO}^{0}$ & 289,428 & 280,932 & 290,640 & 258,735 & 264,411 & 318,465 & 245,154 & 253,159 & 249,514 & 273,292 & 253,023 & 289,423 & 255,720 \\
\hline SE $15^{\circ}$ & 284,727 & 276,459 & 286,365 & 254,793 & 260,416 & 314,420 & 241,222 & 249,245 & 245,636 & 269,306 & 249,504 & 284,309 & 251,744 \\
\hline SE $30^{\circ}$ & 241,205 & 234,293 & 242,780 & 216,076 & 220,541 & 267,203 & 204,203 & 211,121 & 207,890 & 228,132 & 211,173 & 240,929 & 213,442 \\
\hline SE $45^{\circ}$ & 197,506 & 191,895 & 198,670 & 177,090 & 180,488 & 219,626 & 167,110 & 172,747 & 169,967 & 186,710 & 172,755 & 197,405 & 174,755 \\
\hline SE $60^{\circ}$ & 241,747 & 234,673 & 242,150 & 216,787 & 220,608 & 269,609 & 204,258 & 211,057 & 207,823 & 228,320 & 211,077 & 241,801 & 213,699 \\
\hline SE $75^{\circ}$ & 285,907 & 277,346 & 285,364 & 256,574 & 260,461 & 319,893 & 241,470 & 249,083 & 245,390 & 269,899 & 249,530 & 286,260 & 252,533 \\
\hline SE $90^{\circ}$ & 292,108 & 283,557 & 292,189 & 262,310 & 265,582 & 326,559 & 247,106 & 253,545 & 249,942 & 275,338 & 254,597 & 294,597 & 257,155 \\
\hline
\end{tabular}

Table 5: Sum of occurrence of grain components for LLP of size15

\begin{tabular}{|c|c|c|c|c|c|c|c|c|c|c|c|c|c|}
\hline & $\mathrm{T} 1$ & $\mathrm{~T} 2$ & T3 & $\mathrm{T} 4$ & T5 & $\mathrm{T} 6$ & T7 & T8 & T9 & T10 & T11 & T12 & $\mathrm{T} 13$ \\
\hline $\mathrm{SE} 0^{\circ}$ & 330,432 & 320,420 & 331,762 & 295,014 & 301,897 & 363,458 & 280,008 & 288,992 & 285,123 & 312,156 & 289,043 & 330,030 & 292,051 \\
\hline SE $15^{\circ}$ & 325,080 & 315,351 & 326,887 & 290,538 & 297,355 & 358,831 & 275,551 & 284,551 & 280,714 & 307,647 & 285,064 & 324,230 & 287,508 \\
\hline SE $30^{\circ}$ & 281,978 & 273,643 & 283,969 & 252,328 & 257,870 & 312,412 & 238,839 & 246,745 & 243,220 & 266,822 & 247,039 & 281,177 & 249,728 \\
\hline SE $45^{\circ}$ & 236,453 & 229,511 & 238,362 & 211,972 & 216,225 & 263,889 & 200,148 & 206,845 & 203,723 & 223,735 & 207,026 & 235,495 & 209,708 \\
\hline $\mathrm{SE} 60^{\circ}$ & 279,697 & 271,440 & 281,559 & 250,903 & 255,388 & 313,118 & 236,549 & 244,450 & 240,562 & 264,563 & 244,538 & 278,572 & 247,906 \\
\hline SE75 ${ }^{\circ}$ & 326,587 & 316,618 & 326,018 & 293,079 & 297,367 & 366,134 & 275,777 & 284,318 & 280,172 & 308,408 & 284,910 & 326,357 & 288,662 \\
\hline SE $90^{\circ}$ & 333,678 & 323,707 & 333,845 & 299,639 & 303,230 & 373,769 & 282,224 & 289,416 & 285,383 & 314,621 & 290,683 & 335,922 & 293,960 \\
\hline
\end{tabular}

Table 6: Sum of occurrence of grain components for LLP of size 17

\begin{tabular}{|c|c|c|c|c|c|c|c|c|c|c|c|c|c|}
\hline & $\mathrm{T} 1$ & $\mathrm{~T} 2$ & T3 & $\mathrm{T} 4$ & T5 & T6 & $\mathrm{T} 7$ & T8 & T9 & $\mathrm{T} 10$ & $\mathrm{~T} 11$ & $\mathrm{~T} 12$ & T13 \\
\hline $\mathrm{SEO}^{\circ}$ & 370,435 & 358,979 & 371,812 & 330,319 & 338,491 & 407,420 & 314,023 & 324,006 & 319,966 & 350,200 & 324,256 & 369,658 & 327,545 \\
\hline SE $15^{\circ}$ & 364,347 & 353,224 & 366,320 & 325,218 & 333,324 & 402,222 & 308,983 & 318,969 & 314,969 & 345,110 & 319,695 & 363,082 & 322,481 \\
\hline SE $30^{\circ}$ & 318,850 & 309,044 & 321,297 & 285,064 & 291,717 & 354,326 & 270,162 & 278,937 & 275,281 & 301,986 & 279,641 & 317,181 & 282,701 \\
\hline SE $45^{\circ}$ & 273,760 & 265,301 & 276,159 & 245,202 & 250,264 & 306,458 & 231,788 & 239,329 & 235,981 & 259,263 & 239,828 & 271,706 & 243,119 \\
\hline SE $60^{\circ}$ & 319,516 & 309,860 & 321,677 & 286,623 & 291,598 & 358,391 & 270,156 & 279,051 & 274,700 & 302,358 & 279,209 & 317,608 & 283,388 \\
\hline SE $75^{\circ}$ & 366,426 & 355,050 & 365,970 & 328,768 & 333,554 & 411,186 & 309,420 & 318,745 & 314,146 & 346,132 & 319,472 & 365,481 & 323,976 \\
\hline SE $90^{\circ}$ & 374,388 & 362,965 & 374,572 & 336,117 & 340,091 & 419,756 & 316,605 & 324,439 & 320,016 & 353,071 & 325,963 & 376,184 & 329,954 \\
\hline
\end{tabular}


J. Computer Sci., 3 (8): 633-638, 2007

Table 7: Distances between textures using LLP of size 11.

\begin{tabular}{cccccccccccccc}
\hline & $\mathbf{T}_{\mathbf{1}}$ & $\mathbf{T}_{\mathbf{2}}$ & $\mathbf{T}_{\mathbf{3}}$ & $\mathbf{T}_{\mathbf{4}}$ & $\mathbf{T}_{\mathbf{5}}$ & $\mathbf{T}_{\mathbf{6}}$ & $\mathbf{T}_{\mathbf{7}}$ & $\mathbf{T}_{\mathbf{8}}$ & $\mathbf{T}_{\mathbf{9}}$ & $\mathbf{T}_{\mathbf{1 0}}$ & $\mathbf{T}_{\mathbf{1 1}}$ & $\mathbf{T}_{\mathbf{1 2}}$ & $\mathbf{T}_{\mathbf{1 3}}$ \\
\hline $\mathbf{T}_{\mathbf{1}}$ & 0 & 6 & 0 & 48 & 38 & 38 & 91 & 65 & 91 & 16 & 58 & 0 & 53 \\
$\mathbf{T}_{\mathbf{2}}$ & 6 & 0 & 6 & 22 & 16 & 72 & 53 & 33 & 53 & 4 & 28 & 6 & 25 \\
$\mathbf{T}_{\mathbf{3}}$ & 0 & 6 & 0 & 48 & 38 & 38 & 91 & 65 & 91 & 16 & 58 & 0 & 53 \\
$\mathbf{T}_{\mathbf{4}}$ & 48 & 22 & 48 & 0 & 2 & 170 & 7 & 3 & 7 & 10 & 2 & 48 & 1 \\
$\mathbf{T}_{\mathbf{5}}$ & 38 & 16 & 38 & 2 & 0 & 150 & 13 & 5 & 13 & 6 & 4 & 38 & 3 \\
$\mathbf{T}_{\mathbf{6}}$ & 38 & 72 & 38 & 170 & 150 & 0 & 245 & 201 & 245 & 100 & 188 & 38 & 179 \\
$\mathbf{T}_{\mathbf{7}}$ & 91 & 53 & 91 & 7 & 13 & 245 & 0 & 4 & 0 & 33 & 5 & 91 & 6 \\
$\mathbf{T}_{\mathbf{8}}$ & 65 & 33 & 65 & 3 & 5 & 201 & 4 & 0 & 4 & 19 & 1 & 65 & 2 \\
$\mathbf{T}_{9}$ & 91 & 53 & 91 & 7 & 13 & 245 & 0 & 4 & 0 & 33 & 5 & 91 & 6 \\
$\mathbf{T}_{\mathbf{1 0}}$ & 16 & 4 & 16 & 10 & 6 & 100 & 33 & 19 & 33 & 0 & 16 & 16 & 13 \\
$\mathbf{T}_{\mathbf{1 1}}$ & 58 & 28 & 58 & 2 & 4 & 188 & 5 & 1 & 5 & 16 & 0 & 58 & 1 \\
$\mathbf{T}_{\mathbf{1 2}}$ & 0 & 6 & 0 & 48 & 38 & 38 & 91 & 65 & 91 & 16 & 58 & 0 & 53 \\
$\mathbf{T}_{\mathbf{1 3}}$ & 53 & 25 & 53 & 1 & 3 & 179 & 6 & 2 & 6 & 13 & 1 & 53 & 0 \\
\hline
\end{tabular}

Table 8: Distances between textures using LLP of size 13.

\begin{tabular}{cccccccccccccc}
\hline & $\mathbf{T}_{\mathbf{1}}$ & $\mathbf{T}_{\mathbf{2}}$ & $\mathbf{T}_{\mathbf{3}}$ & $\mathbf{T}_{\mathbf{4}}$ & $\mathbf{T}_{\mathbf{5}}$ & $\mathbf{T}_{\mathbf{6}}$ & $\mathbf{T}_{\mathbf{7}}$ & $\mathbf{T}_{\mathbf{8}}$ & $\mathbf{T}_{\mathbf{9}}$ & $\mathbf{T}_{\mathbf{1 0}}$ & $\mathbf{T}_{\mathbf{1 1}}$ & $\mathbf{T}_{\mathbf{1 2}}$ & $\mathbf{T}_{\mathbf{1 3}}$ \\
\hline $\mathbf{T}_{\mathbf{1}}$ & 0 & 6 & 1 & 48 & 38 & 65 & 114 & 84 & 84 & 13 & 84 & 0 & 70 \\
$\mathbf{T}_{\mathbf{2}}$ & 6 & 0 & 7 & 24 & 16 & 107 & 72 & 48 & 48 & 3 & 48 & 6 & 38 \\
$\mathbf{T}_{\mathbf{3}}$ & 1 & 7 & 0 & 55 & 43 & 60 & 123 & 91 & 91 & 16 & 91 & 1 & 77 \\
$\mathbf{T}_{\mathbf{4}}$ & 48 & 24 & 55 & 0 & 2 & 223 & 16 & 6 & 6 & 13 & 6 & 48 & 4 \\
$\mathbf{T}_{\mathbf{5}}$ & 38 & 16 & 43 & 2 & 0 & 199 & 22 & 10 & 10 & 7 & 10 & 38 & 6 \\
$\mathbf{T}_{\mathbf{6}}$ & 65 & 107 & 60 & 223 & 199 & 0 & 349 & 295 & 295 & 132 & 295 & 65 & 267 \\
$\mathbf{T}_{\mathbf{7}}$ & 114 & 72 & 123 & 16 & 22 & 349 & 0 & 4 & 4 & 53 & 4 & 114 & 6 \\
$\mathbf{T}_{\mathbf{8}}$ & 84 & 48 & 91 & 6 & 10 & 295 & 4 & 0 & 0 & 33 & 0 & 84 & 2 \\
$\mathbf{T}_{\mathbf{9}}$ & 84 & 48 & 91 & 6 & 10 & 295 & 4 & 0 & 0 & 33 & 0 & 84 & 2 \\
$\mathbf{T}_{\mathbf{1 0}}$ & 13 & 3 & 16 & 13 & 7 & 132 & 53 & 33 & 33 & 0 & 33 & 13 & 25 \\
$\mathbf{T}_{\mathbf{1 1}}$ & 84 & 48 & 91 & 6 & 10 & 295 & 4 & 0 & 0 & 33 & 0 & 84 & 2 \\
$\mathbf{T}_{\mathbf{1 2}}$ & 0 & 6 & 1 & 48 & 38 & 65 & 114 & 84 & 84 & 13 & 84 & 0 & 70 \\
$\mathbf{T}_{\mathbf{1 3}}$ & 70 & 38 & 77 & 4 & 6 & 267 & 6 & 2 & 2 & 25 & 2 & 70 & 0 \\
\hline
\end{tabular}

Table 9: Distances between textures using LLP of size 15.

\begin{tabular}{|c|c|c|c|c|c|c|c|c|c|c|c|c|c|}
\hline 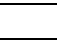 & $\mathrm{T}_{1}$ & $T_{2}$ & $T_{3}$ & $\mathrm{~T}_{4}$ & $T_{5}$ & $T_{6}$ & $\mathrm{~T}_{7}$ & $\mathrm{~T}_{8}$ & $\mathrm{~T}_{9}$ & $T_{10}$ & $T_{11}$ & $\mathrm{~T}_{12}$ & $T_{13}$ \\
\hline$T_{1}$ & 0 & 7 & 0 & 77 & 48 & 72 & 148 & 116 & 130 & 25 & 107 & 2 & 91 \\
\hline $\mathrm{T}_{2}$ & 7 & 0 & 7 & 38 & 19 & 123 & 91 & 67 & 77 & 6 & 60 & 9 & 48 \\
\hline $\mathbf{T}_{3}$ & 0 & 7 & 0 & 77 & 48 & 72 & 148 & 116 & 130 & 25 & 107 & 2 & 91 \\
\hline $\mathrm{T}_{4}$ & 77 & 38 & 77 & 0 & 5 & 295 & 13 & 5 & 7 & 16 & 4 & 77 & 2 \\
\hline $\mathbf{T}_{5}$ & 48 & 19 & 48 & 5 & 0 & 236 & 28 & 16 & 22 & 5 & 13 & 50 & 7 \\
\hline$T_{6}$ & 72 & 123 & 72 & 295 & 236 & 0 & 424 & 368 & 392 & 179 & 353 & 72 & 323 \\
\hline$T_{7}$ & 148 & 91 & 148 & 13 & 28 & 424 & 0 & 4 & 2 & 53 & 5 & 150 & 7 \\
\hline $\mathbf{T}_{8}$ & 116 & 67 & 116 & 5 & 16 & 368 & 4 & 0 & 2 & 35 & 1 & 116 & 3 \\
\hline$T_{9}$ & 130 & 77 & 130 & 7 & 22 & 392 & 2 & 2 & 0 & 43 & 3 & 130 & 5 \\
\hline$T_{10}$ & 25 & 6 & 25 & 16 & 5 & 179 & 53 & 35 & 43 & 0 & 30 & 27 & 22 \\
\hline $\mathrm{T}_{11}$ & 107 & 60 & 107 & 4 & 13 & 353 & 5 & 1 & 3 & 30 & 0 & 109 & 2 \\
\hline $\mathrm{T}_{12}$ & 2 & 9 & 2 & 77 & 50 & 72 & 150 & 116 & 130 & 27 & 109 & 0 & 93 \\
\hline $\mathrm{T}_{13}$ & 91 & 48 & 91 & 2 & 7 & 323 & 7 & 3 & 5 & 22 & 2 & 93 & 0 \\
\hline
\end{tabular}

Table 10: Distances between textures using LLP of size 17.

\begin{tabular}{|c|c|c|c|c|c|c|c|c|c|c|c|c|c|}
\hline & $\mathrm{T}_{1}$ & $\mathbf{T}_{2}$ & $\mathrm{~T}_{3}$ & $\mathbf{T}_{4}$ & $T_{5}$ & $\mathbf{T}_{6}$ & $\mathrm{~T}_{7}$ & $\mathbf{T}_{8}$ & $\mathrm{~T}_{9}$ & $\mathbf{T}_{10}$ & $\mathbf{T}_{11}$ & $\mathbf{T}_{12}$ & $\mathrm{~T}_{13}$ \\
\hline$T_{1}$ & 0 & 6 & 2 & 72 & 65 & 114 & 188 & 132 & 161 & 22 & 123 & 1 & 114 \\
\hline$T_{2}$ & 6 & 0 & 10 & 38 & 33 & 168 & 130 & 84 & 107 & 6 & 77 & 9 & 70 \\
\hline $\mathbf{T}_{3}$ & 2 & 10 & 0 & 84 & 77 & 100 & 208 & 148 & 179 & 28 & 139 & 3 & 130 \\
\hline $\mathbf{T}_{4}$ & 72 & 38 & 84 & 0 & 1 & 362 & 28 & 10 & 19 & 16 & 7 & 79 & 6 \\
\hline $\mathbf{T}_{5}$ & 65 & 33 & 77 & 1 & 0 & 347 & 33 & 13 & 22 & 13 & 10 & 72 & 7 \\
\hline $\mathbf{T}_{6}$ & 114 & 168 & 100 & 362 & 347 & 0 & 590 & 488 & 541 & 232 & 469 & 105 & 452 \\
\hline$T_{7}$ & 188 & 130 & 208 & 28 & 33 & 590 & 0 & 6 & 3 & 84 & 7 & 199 & 10 \\
\hline $\mathbf{T}_{8}$ & 132 & 84 & 148 & 10 & 13 & 488 & 6 & 0 & 3 & 48 & 1 & 143 & 2 \\
\hline $\mathrm{T}_{9}$ & 161 & 107 & 179 & 19 & 22 & 541 & 3 & 3 & 0 & 67 & 4 & 172 & 5 \\
\hline$T_{10}$ & 22 & 6 & 28 & 16 & 13 & 232 & 84 & 48 & 67 & 0 & 43 & 27 & 38 \\
\hline$T_{11}$ & 123 & 77 & 139 & 7 & 10 & 469 & 7 & 1 & 4 & 43 & 0 & 132 & 1 \\
\hline$T_{12}$ & 1 & 9 & 3 & 79 & 72 & 105 & 199 & 143 & 172 & 27 & 132 & 0 & 123 \\
\hline$T_{13}$ & 114 & 70 & 130 & 6 & 7 & 452 & 10 & 2 & 5 & 38 & 1 & 123 & 0 \\
\hline
\end{tabular}


where $d_{i}=20$

The study of distance function Table 8 of LLP $1 \times 13$ depicts the following texture classes for a distance threshold value of 20 .

$$
\begin{aligned}
& \mathrm{C} 1=\left\{\mathrm{T}_{1}, \mathrm{~T}_{2}, \mathrm{~T}_{3}, \mathrm{~T}_{10}, \mathrm{~T}_{12}\right\} \\
& \mathrm{C} 2=\left\{\mathrm{T}_{4}, \mathrm{~T}_{7}, \mathrm{~T}_{8}, \mathrm{~T}_{9}, \mathrm{~T}_{11}, \mathrm{~T}_{13}\right\} \\
& \mathrm{C} 3=\left\{\mathrm{T}_{5}\right\} \\
& \mathrm{C} 4=\left\{\mathrm{T}_{6}\right\} .
\end{aligned}
$$

Here texture $T_{5}$ is not placed in class $C 2$ because $T_{5}$ differs with $T_{7}$, a distance threshold value greater than 20. However $\mathrm{T}_{5}$ differs with all other textures of $\mathrm{C} 2$ by a distance factor less than 20.Therefore the other way of writing class $\mathrm{C} 3$ is

$\mathrm{C} 3=\left\{\mathrm{T}_{1}, \mathrm{~T} 5, \mathrm{~T} 8, \mathrm{~T} 9, \mathrm{~T} 11, \mathrm{~T} 13\right\}$.

The clear observation of distance function in Table 9 of LLP $1 \times 15$ depicts the following texture classes for a distance threshold value of 20 .

$\mathrm{C} 1=\left\{\mathrm{T}_{1}, \mathrm{~T}_{2}, \mathrm{~T} 3, \mathrm{~T}_{12}\right\}$

$\mathrm{C} 2=\left\{\mathrm{T}_{4}, \mathrm{~T}_{7}, \mathrm{~T}_{8}, \mathrm{~T}_{9}, \mathrm{~T}_{11}, \mathrm{~T}_{13}\right\}$

$\mathrm{C} 3=\left\{\mathrm{T}_{5}\right\}$ or $\left\{\mathrm{T}_{4}, \mathrm{~T}_{5}, \mathrm{~T}_{8}, \mathrm{~T}_{9}, \mathrm{~T}_{11}, \mathrm{~T}_{13}\right\}$

$\mathrm{C} 4=\left\{\mathrm{T}_{6}\right\}$

$\mathrm{C} 5=\left\{\mathrm{T}_{10}\right\}$.

The examination of distance function Table 10 of LLP $1 \times 17$ depicts the following texture classes for a distance threshold value of 20

$\mathrm{C} 1=\left\{\mathrm{T}_{1}, \mathrm{~T}_{2}, \mathrm{~T}_{3}, \mathrm{~T}_{12}\right\}$

$\mathrm{C} 2=\left\{\mathrm{T}_{4}, \mathrm{~T}_{8}, \mathrm{~T}_{9}, \mathrm{~T}_{11}, \mathrm{~T}_{13}\right\}$

$\mathrm{C} 3=\left\{\mathrm{T}_{5}\right\}$ or $\left\{\mathrm{T}_{4}, \mathrm{~T}_{5}, \mathrm{~T}_{8}, \mathrm{~T}_{11}, \mathrm{~T}_{13}\right\}$

$\mathrm{C} 4=\left\{\mathrm{T}_{7}\right\}$ or $\left\{\mathrm{T}_{7}, \mathrm{~T}_{8}, \mathrm{~T}_{9}, \mathrm{~T}_{11}, \mathrm{~T}_{13}\right\}$

$\mathrm{C} 5=\left\{\mathrm{T}_{10}\right\}$

$\mathrm{C} 6=\left\{\mathrm{T}_{6}\right\}$.

\section{CONCLUSIONS}

The analysis about distance functions of LLP concludes that the number of overlapping texture classes will be more by increasing the linear element size. The texture groups or classes are very concise by LLP of size $1 \times 11$. The sum of the grain components of large linear patterns $1 \times 11,1 \times 13,1 \times 15$ and $1 \times 17$ as listed in the Tables 3,4,5 and 6 respectively indicates a decreasing trend from $0^{0}$ to $45^{\circ}$ and an increased trend from $45^{\circ}$ to $90^{\circ}$ of grain components. This clearly reflects the geometrical property on linearity that is reflection about the line $\mathrm{X}=\mathrm{Y}$. From this the present paper concludes that, it is not necessary to compute the orientations of linear patterns from $90^{\circ}$ to $360^{\circ}$, as they can be counted merely by reflection of $\mathrm{X}$ axis and $\mathrm{Y}$ axis.

\section{ACKNOWLEDGEMENTS}

The authors would like to express their cordial thanks to K.V.V. Satya Narayana Raju, Chairman, Chaitanya
Institutions and K. Sashi Kiran Varma, Secretary, GIET,Rajahmundry for providing Research facilities.

\section{REFERENCES}

1. Hawkins J.K, 1969, Textural Properties for Pattern Recognition, Picture Processing and Psychopictories (editors B.Lipkin and A.Rosenfeld), Academic Press, New York.

2. R. W. Conners and C. A. Harlow, 1980. A Theoretical Comparison Texture Algorithms, IEEE Transactions on Pattern Analysis and Machine Intelligence, 2, pp. 204-222.

3. A. Laine and J. Fan, 1993. Texture classification by wavelet packet signatures, IEEE Trans. on PAMI, 15(11), pp. 1186-1190.

4. A. Bovik, M. Clark, W. S. Geisler, 1990. Multichannel Texture Analysis Using Localized Spatial Filters, IEEE Transactions on Pattern Analysis and Machine Intelligence, 12(1), pp. 5573.

5. A. K. Jain and F. Farrokhnia, 1991.Unsupervised texture segmentation using Gabor filters, Pattern Recognition, 24(12), pp. 1167-1186.

6. M. Unser, M. Eden, 1989. Multiresolution feature extraction and selection for texture segmentation, IEEE Transactions on Pattern Analysis and Machine Intelligence, 11, pp. 717- 728.

7. Hiremath, P. S. and Shivashankar, S, 2006.Texture classification using Wavelet Packet Decomposition, ICGSTs GVIP Journal, 6(2), pp. 77-80.

8. S.Arivazhagan, L.Ganesan, 2003. Texture classification using wavelet transforms, Pattern Recognition Letters, 24, pp. 1513-1521.

9. R. M. Haralick, K. Shanmugam, I. Dinstein, 1973.Texture features for image classification, IEEE Trans. System Man Cybernat, 8 (6), pp. 610621.

10. E. Montiel, A. S. Aguado, M. S. Nixon, 2005. Texture Classification via conditional histograms, Pattern Recognition Letters, 26, pp. 1740-1751.

11. Matheron, 1975.Random Sets and Integral Geometry, Wiley, New York.

12. J.Serra, 1982. Image Analysis and Mathematical Morphology, Academic Press, London.

13. E.Bresenham, 1965. Algorithm for Computer Control of a Digital Plotter, IBS Systems Journal, 4(1): 25- 30 . 